\title{
Sign Identification of Nonlinear Refractive Index of Colloidal Nanoparticles by Moiré Deflectometry Technique
}

\author{
A. Granmayeh Rad \\ granmayeh@riau.ac.ir \\ K. Madanipour
}

\author{
Plasma Physics Research Center, Science and Research branch, Islamic Azad University (IAU), Tehran, \\ Iran \\ Optical Measurement Central Lab, Amirkabir University of Technology, Tehran, Iran
}

Department of physics, University of Tehran, Tehran, Iran

Physics Department, Science Faculty, Kashan University, Kashan, Iran

In this paper, a visual rapid technique is presented for the sign identification of nonlinear refractive index of colloidal nanoparticles based on non-scanning Moiré deflectometry technique. In this method two lasers are used, one as a pump laser beam which causes thermal nonlinear effects in the sample and the second one is used as a probe beam laser which allows us to monitor these effects by Moiré deflectometry technique. The gradient of the nonlinear refractive index produced by the interaction of the pump laser, generates a cylindrical lens in the sample. The concave and convex lenses are produced as a result of negative and positive nonlinear refractive index respectively. Ceometrical and experimental investigations show the Moiré fringes are deflected in two different directions by these lenses. By observing the shape of deflected moire fringes, we can determine the sign of nonlinear refractive index and there will be no need for calibration or complicated calculations. This technique was applied for identification of nonlinear refractive index of $\mathrm{Au}^{2}$ and Tio $\mathrm{C}_{2}$ colloidal nanoparticles, under $47 \mathrm{~mW}$ second harmonic of $\mathrm{Nd}$ :YAC laser illumination. The sign of nonlinear refractive index of colloidal Au and Tio 2 nanoparticles were observed to be negative and positive respectively.

[DOI: http://dx.doi.org/10.2971/jeos.2012.12034]

Keywords: Moiré deflectometry, nonlinear refractive index, nanoparticles, sign identification

\section{INTRODUCTION}

The measurement of nonlinear optical parameters of colloidal metallic nanoparticles has recently drawn a lot of attentions because of their fast nonlinear optical response and high nonlinearity ability [1]. Colloidal metallic solutions are frequently used in the design of optical instruments and photonic limiters because of their photo-induced nonlinear properties $[2,3]$. There are two standard and usual methods for sign identification of nonlinear refractive index of nanoparticles, Z-scan [4]-[7] and Moiré deflectometry techniques [8]-[13]. In Z-scan method the scanning starts from a distance far away from the focus (negative $\mathrm{z}$ ) and when the sample is brought closer to the focus, the beam irradiance increases leading to self-lensing in the sample. In a negative nonlinearity a negative self-lensing prior to focus tends to collimate the beam and reduce the diffraction leading to a smaller beam at the aperture and an increased transmittance. As the sample crosses the focal plane to the right (positive $\mathrm{z}$ ) the diffraction of the beam will be augmented and the aperture transmittance will be reduced due to the same self-defocusing effect. A pre-focal transmittance maximum (peak) and a post-focal transmittance minimum (valley) are, therefore, the representative of the negative sign of nonlinear refractive index. This is apparent from the peak-valley configuration. The Z-scan signature of a positive nonlinearity, following the same analogy, will give rise to an opposite valley-peak configuration. Z-scan method requires sensitive and calibrated detector which makes the accuracy of measurement dependent on the accuracy of detector's response. By using Moiré deflectomety technique, instead of drawing a diagram based on the $\mathrm{z}$ dependence of the transmitted fluence, the nonlinear refractive index of materials and its sign is obtained by analyzing the Moiré fringes patterns [14]. It has been shown that Moiré methods applications are simpler and more robust than other methods [15]. Recently, sign identification of third nonlinear refractive index of materials by Moiré deflectometry technique, is obtained by observing Moiré fringes sizes and the Moiré fringes spacing curves [16, 17]. This technique solves the problem of highly calibrated detectors but still the need to use a scanner which is highly sensitive to movements exists.

In the presented method in this paper which is based on Moiré deflectometry technique not only highly sensitive calibrated detectors are not required but also the problem of scanning is solved too and there is no need of scanning excitation beam. In this work two laser beams have been used to sign identify the nonlinear refractive index of $\mathrm{Au}$ and $\mathrm{Tio}_{2}$ nanoparticles suspended in water. The basic idea is to emit a laser beam with a high intensity as a pump beam, to the sample. Emitting this beam causes nonlinear effects in the sample which will lead to 


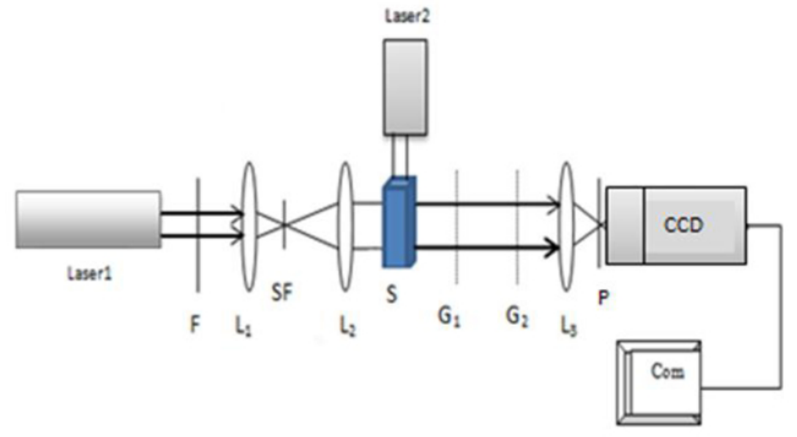

FIC. 1 The experimental set up of Moiré deflectometry for sign identifying nonlinear refractive index, (a): Laser1; $15 \mathrm{~mW}$ He-Ne laser, Laser2; $47 \mathrm{~mW}$ green laser, F; Filter, SF; spatial filter, $G_{1}, G_{2}$; grating, $L_{1}, L_{2}, L_{3}$; lenses with the focal lengths of $f_{1}=50 \mathrm{~mm}$, $f_{2}=150 \mathrm{~mm}, f_{3}=250 \mathrm{~mm}, \mathrm{~S}$ sample, $\mathrm{P}$; the pinhole, CCD; the camera, Com; the computer.

changes in the refractive index in the sample environment. An expanded beam of low intensity will be emitted perpendicularly on the pump beam. This probe beam can then determine the sign of nonlinear refractive index with no need for numerical calculations.

\section{EXPERIMENTAL SET UP}

Figure 1 shows the Moiré deflectometry experimental set-up which was used to measure the nonlinear refractive index of $\mathrm{Au}$ nanoparticles suspended in pure water. The probe laser beam (Laser.1) is a $15 \mathrm{~mW} \mathrm{He-Ne} \mathrm{laser} \mathrm{beam} \mathrm{with} \mathrm{wavelength}$ $\lambda=632.8 \mathrm{~nm}$. To avoid saturation of the fringe images, we used a natural filter $F$, so the power is reduced. The laser beam is focused by the lens $\mathrm{L}_{1}$. After passing through the spatial filter SF, the beam is collimated by lens $\mathrm{L}_{2}$. The focal length of lenses $L_{1}$ and $L_{2}$ are 50 and $150 \mathrm{~mm}$, respectively. Two similar Ronchi grating $G_{1}$ and $G_{2}$ with pitch of $0.1 \mathrm{~mm}$ are used for Moire deflectometry. The grating $G_{2}$ is located in the fifth Talbot distance of grating $\mathrm{G}_{1}$.

To generate nonlinear effects in the nanoparticle sample $S$, a second harmonic Nd:YAG green laser (Laser.2) with the wavelength $\lambda=532 \mathrm{~nm}$ and the power of $47 \mathrm{~mW}$ was used. This pump beam is perpendicular to the probe beam.

The lens $\mathrm{L}_{3}$ with focal length of $250 \mathrm{~mm}$ was placed at the back of second grating $G_{2}$. The pinhole $P$ was used at focal plane of Lens $\mathrm{L}_{3}$ to filter the first diffraction order of gratings. The Moiré fringes patterns were captured by a CCD camera and then transferred to computer (Com).

\section{THEORY}

Interaction of the pump laser and colloidal nanoparticles will result in thermal nonlinear effect and this in turn will change the refractive index of interaction zone is changed. The intensity-dependent refractive index $n$, is defined as [18]:

$$
n=n_{0}+\Delta n=n_{0}+n_{2} I
$$
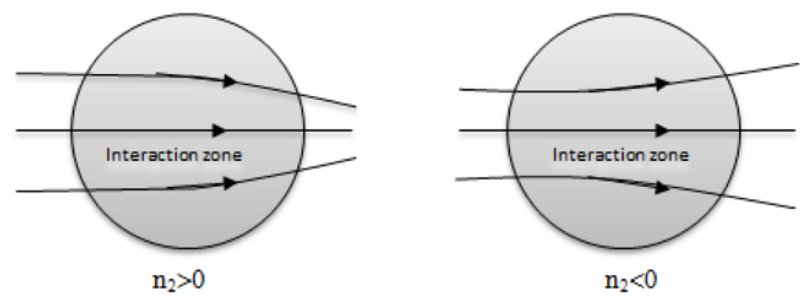

FIG. 2 Interaction zone between guide laser beam and nonlinear environment, (a): A convex lens as a result of positive refractive index. (b): A concave lens as a result of negative refractive index.

Where $n_{0}$ is the first refractive index, $n_{2}$ the second order of refractive index and Ithe intensity of the incident pump beam which causes nonlinear effects. Although the intensity profile of laser is Gaussian, we suppose that the intensity of pump beam is uniform. This assumption does not alter the results. The difference between refractive index of interaction zone and other area of sample creates a cylindrical lens which is shown in Figure 2.

According to lens-makers formula, focal length of this lens can be written as [19]:

$$
\frac{1}{f}=\left(n-n_{0}\right)\left[\frac{1}{R_{1}}-\frac{1}{R_{2}}\right] .
$$

Where $R_{1}$ and $R_{2}$ are the first and second radius of a lens respectively and equal to the pump laser beam radius $\mathrm{R}$. By using Eq. (1) with (2), the focal length of cylindrical lens created by the nonlinear effects will be:

$$
\mathrm{f}=\frac{\mathrm{R}}{2 \mathrm{n}_{2} \mathrm{I}} .
$$

Eq. (3) shows that concave or convex lenses are generated as a result of negative or positive nonlinear refractive indexes of sample, respectively. Convergence or divergence of probe beam illuminated on the generated cylindrical lens will determine the sign of nonlinear refractive index. The convergence of the beam corresponds to positive $\left(n_{2}>0\right)$ and divergence of the beam corresponds to negative $\left(n_{2}<0\right)$ nonlinear refractive index.

As shown in Figure 1, to identify the sign of nonlinear refractive index using the Moiré technique, a low intensity wide collimated beam is illuminated on the sample perpendicular to the pump beam. The probe beam is deflected by passing through the pump beam. This deflection is caused by the generated cylindrical lens.

By choosing the appropriate coordinates as shown in Figures 3 to 6 and rotating the grating along the $Z$ axes, we can observe the effect of rotation of the first grating on the deflection moiré fringe patterns. Figures 3 and 4 show the effect of change of first grating angle on the deflection moiré fringe patterns of a sample with negative refractive index while Figures 5 and 6 show this effect for a sample with positive refractive index.

For a sample with negative refractive index By rotating of the first grating angel, $\theta_{G 1}$, to the positive direction(Figure 3(a)) the moiré fringe patterns are also deflected to the positive direction as shown in Figure 3(b) and the deflection patterns 


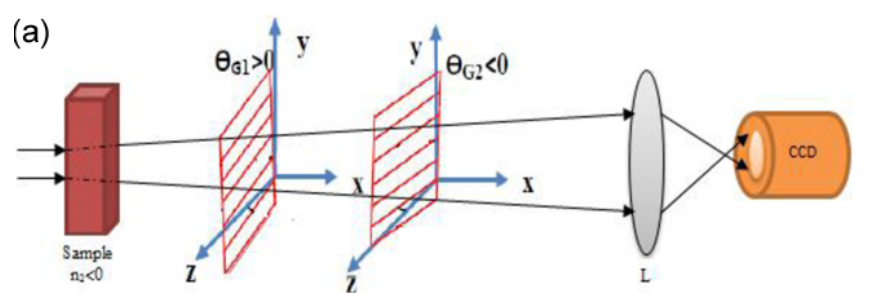

(b)

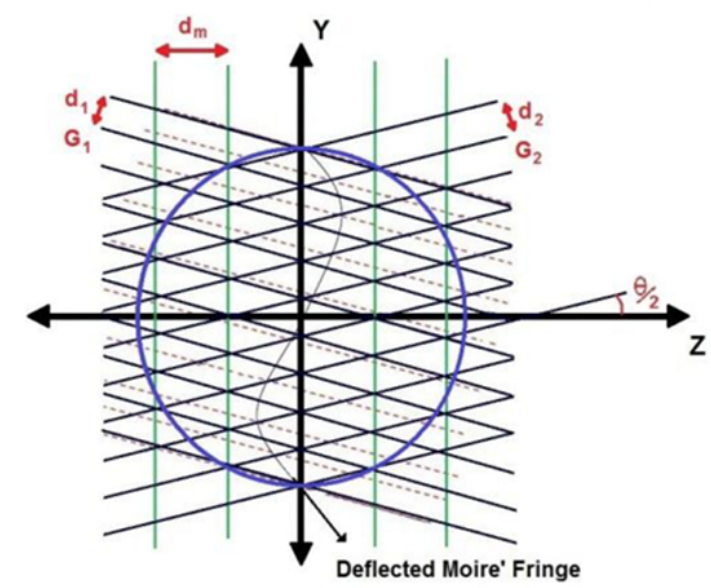

FIG. 3 The experimental setup for $n_{2}<0, \theta_{G 1}>0$, (b) deflection of moiré pattern; the red dash lines show the movement of the grating vector image, in which $\mathrm{G}_{i}$ is grating, $\mathrm{L}$ is lens and $d_{i}$ and $d_{m}$ are the pitches of grating and Moiré fringes, respectively.

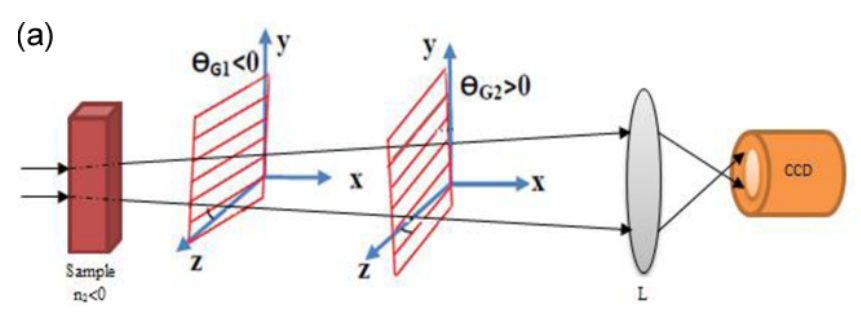

(b)

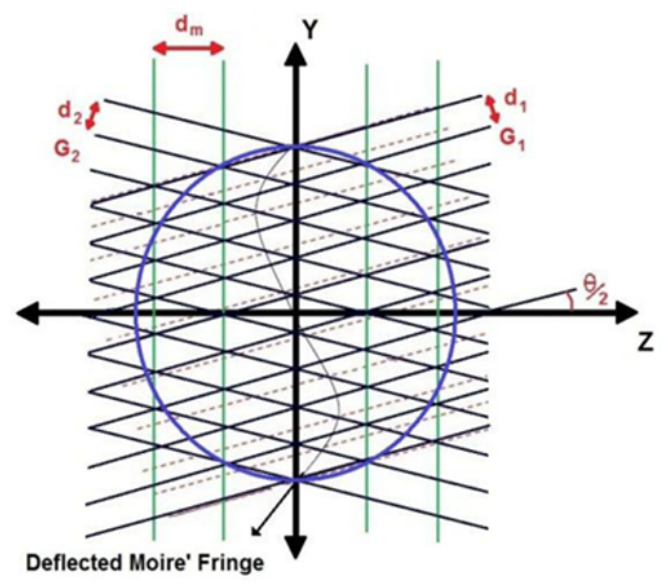

FIG. 4 The experimental setup for $n_{2}<0, \theta_{G 1}<0$, (b) deflection of moiré pattern; the red dash lines show the movement of the grating vector image, in which in which $\mathrm{C}_{i}$ is grating, $\mathrm{L}$ is lens and $d_{i}$ and $d_{m}$ are the pitches of grating and Moiré fringes, respectively.

happen in an opposite direction when $\theta_{G 1}<0$, (Figures 4 (a) and $4(\mathrm{~b}))$.

On the other hand for materials with positive refractive index by rotating the first grating in the positive direction, $\theta_{G 1}>0$, (Figure 5(a)), the moiré fringes are deflected in the positive

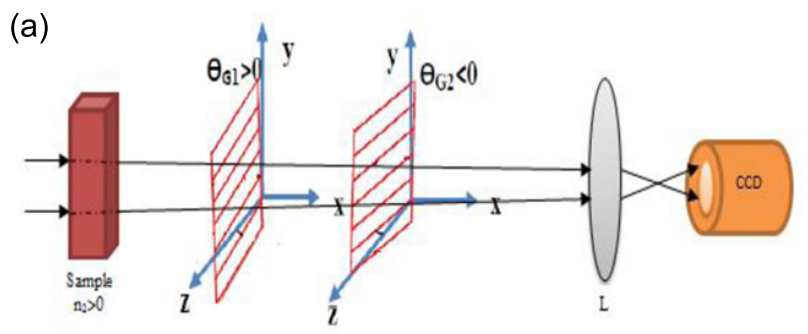

(b)

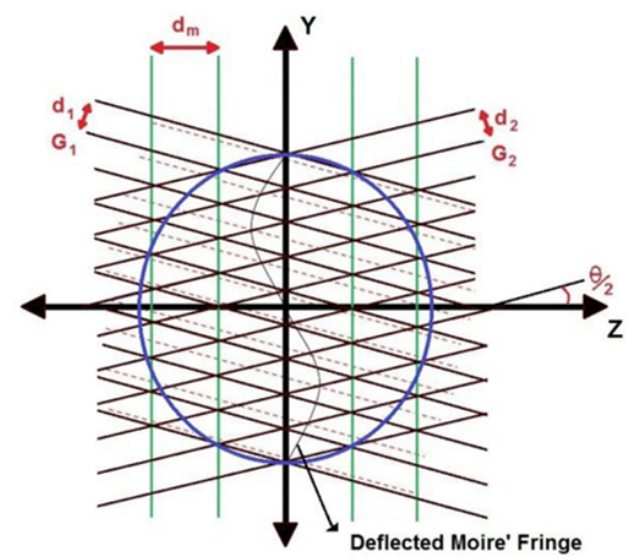

FIC. 5 The experimental setup for $n_{2}>0, \theta_{G 1}>0$, (b)deflection of moiré pattern, the red dash lines show the movement of the grating vector image, in which $\mathrm{C}_{i}$ is grating, $\mathrm{L}$ is lens and $d_{i}$ and $d_{m}$ are the pitches of grating and Moiré fringes, respectively.

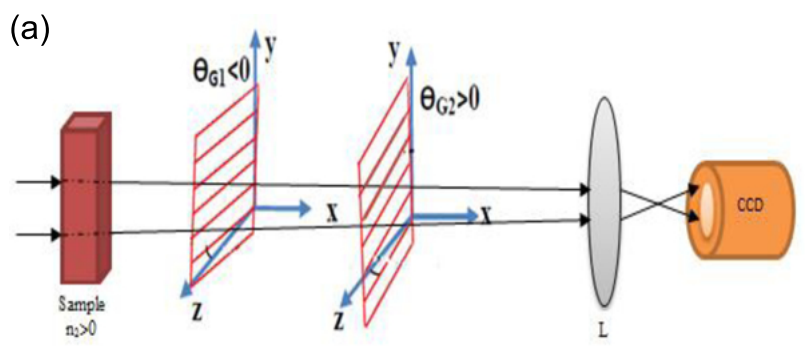

(b)

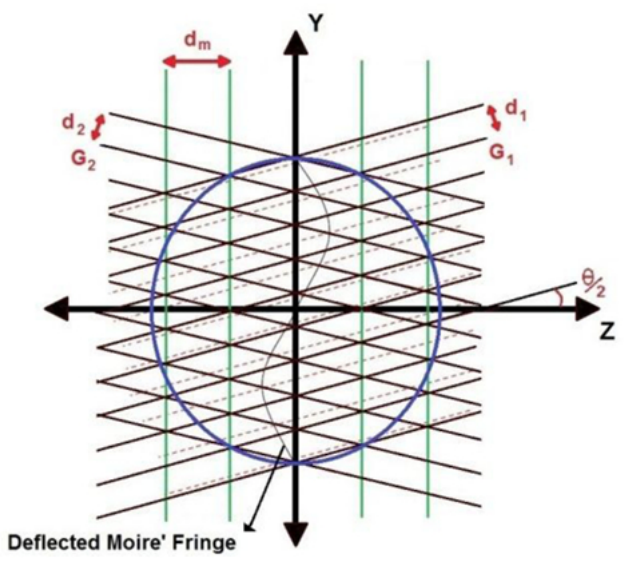

FIG. 6 The experimental setup for $n_{2}>0, \theta_{G 1}<0$, (b)deflection of moiré pattern, the red dash lines show the movement of the grating vector image, in which $\mathrm{G}_{i}$ is grating, $\mathrm{L}$ is lens and $d_{i}$ and $d_{m}$ are the pitches of grating and Moiré fringes, respectively.

direction, (Figures 5(a) and 5(b)), and vice versa, (Figures 6(a) and $6(b))$.

After setting the first grating direction $\theta_{G 1}$, and by observing deflected moiré fringes images, the sign of nonlinear refractive index can be determined. If the sample is concave $\left(n_{2}<0\right)$, the image of grating lines will recede from the center. On the other hand if the sample is convex $\left(n_{2}>0\right)$, the image of grating 

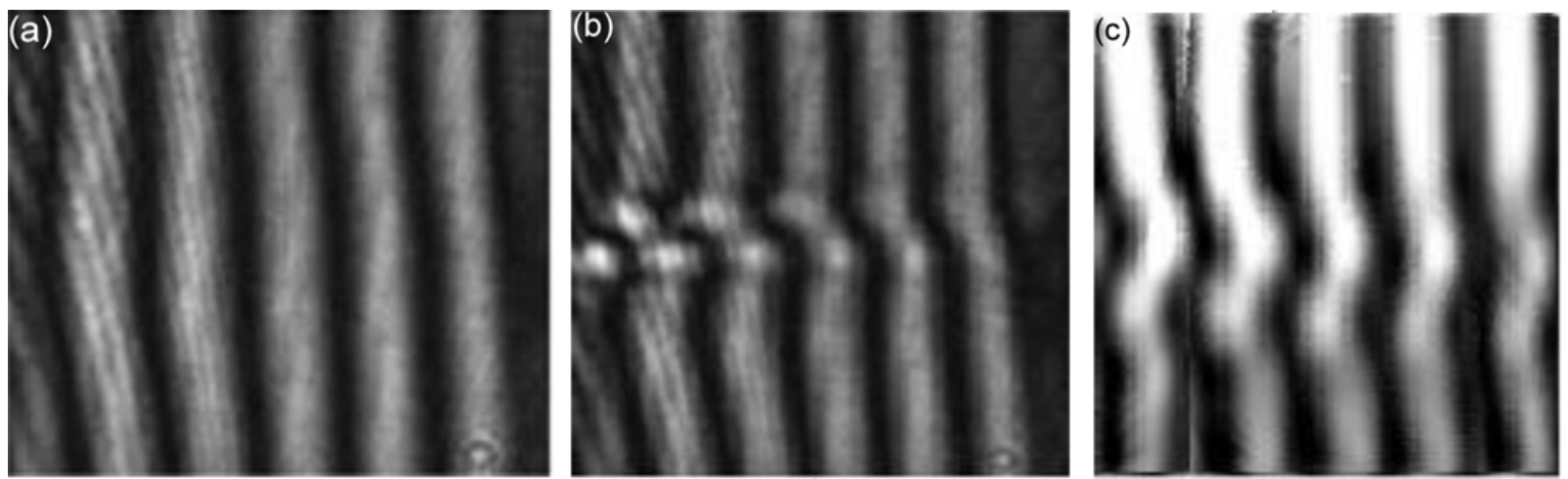

FIG. 7 The deflection of Moiré fringes patterns of Au nanopraticles caused by producing refractive index gradient, (a) the Moiré fringes before deflection, (b) the Moiré fringes after deflection, $\theta_{G 1}<0$, (c) the Moiré fringes after deflection, $\theta_{G 1}>0$.
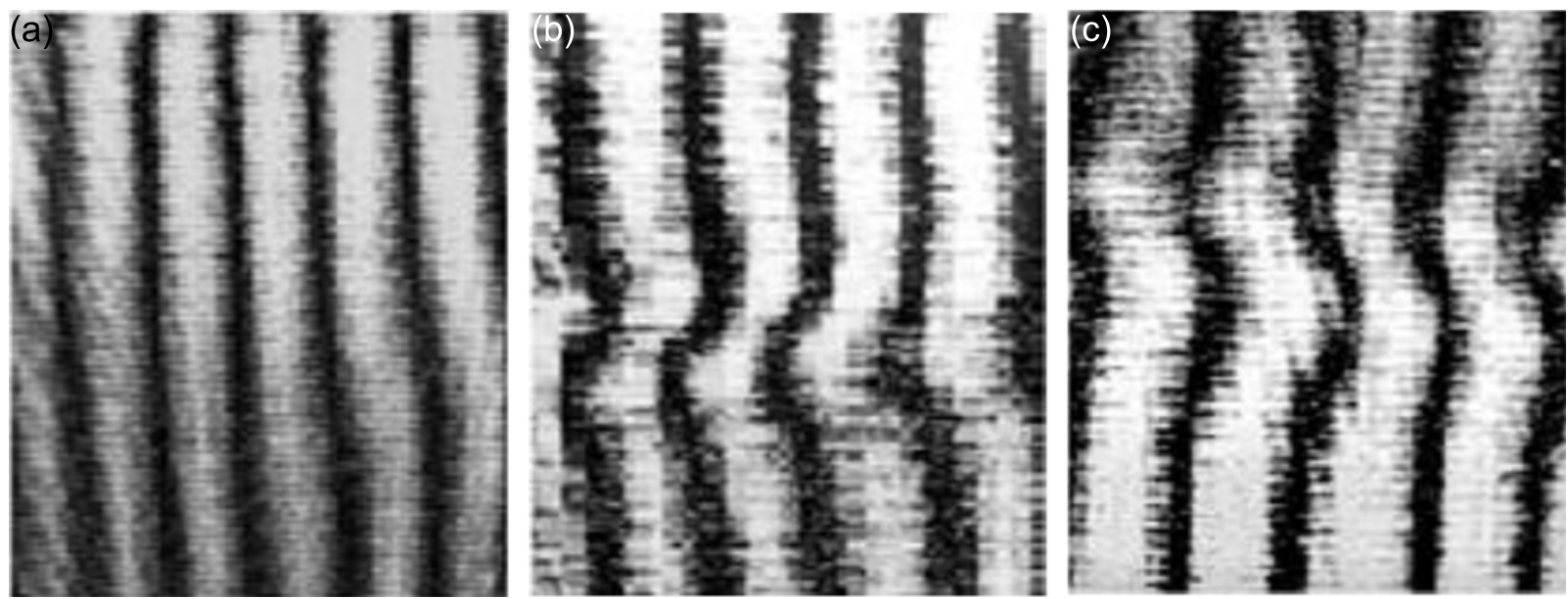

FIG. 8 The deflection of Moiré fringes patterns of $\mathrm{TiO}_{2}$ nanoparticles caused by producing refractive index gradient, (a) the Moiré fringes before deflection, (b) the Moiré fringes after deflection, $\theta_{G 1}<0$, (c) the Moiré fringes after deflection, $\theta_{G 1}>0$.

lines will approach to the center. There are no deflection in the border and the center of the sample in $\mathrm{Y}$ direction (blue circle in Figure 3(b), Figure 4(b), Figure 5(b), Figure 6(b)), and maximum deflections happen in the region between borders and center in $\mathrm{Y}$ direction.

\section{EXPERIMENTAL RESULTS AND DISCUSSIONS}

We have examined the Moiré deflectometry technique for measuring the nonlinear refractive index in colloidal $\mathrm{Au}$ and $\mathrm{TiO}_{2}$ nanoparticles. To identify the sign of nonlinear refractive index of the sample, the experiment was set up as shown in Figure 1, a $15 \mathrm{~mW}$ He-Ne laser beam has been used as a guide beam, which is expanded and collimated by lens $\mathrm{L}_{1}, \mathrm{~L}_{2}$ and a spatial filter and then passes through nanoparticles, which is in a Quartz cell with $10 \mathrm{~mm}$ thickness. As the laser beam passes through grating $G_{1}$ and $G_{2}$, Moiré fringe patterns are projected on a CCD camera by lens $\mathrm{L}_{3}$ and recorded by a computer (Figures 7(a) and 8(a)). A $47 \mathrm{~mW}$ second harmonic of Nd:YAG laser is used as a pump, the beam is emitted to the sample. After generating the thermal gradient in the colloidal nanoparticles, the deflection of Moiré fringes will appear. As shown in Figure 7, the deflected Moiré fringes were determined for Au nanoparticles. By changing the direction of first grating angle for $\theta_{G 1}<0$ and $\theta_{G 1}>0$, the deflected Moiré fringes change patterns as shown in Figure 7(b) and 7(c). Finally by knowing the direction of deflected Moiré fringes and the direction of rotation of first grating angle, the sing of nonlinear refractive index of $\mathrm{Au}$ nanoparticles was found to be negative.

Similarly for $\mathrm{Tio}_{2}$ nanoparticles the deflected Moiré fringes were observed by changing the direction of first grating angle. The results for $\theta_{G 1}<0$ and $\theta_{G 1}>0$ are shown, Figure $8(b)$ and 8 (c) and it was found that $\mathrm{Tio}_{2}$ nanoparticles have a positive nonlinear refractive index.

\section{CONCLUSION}

As shown in this paper, a simple method based on the Moiré deflectometry technique can be used to identify the sign of nonlinear refractive thermal index, caused by the interaction of a laser with the colloidal gold nanoparticles and titanium dioxide nanoparticles in water solution.

The proposed Moiré deflectometry technique is a nonscanning method, and the sign of refractive index can be achieved immediately and in the real time. By visual investigation of moiré fringes deflection, the sign of nonlinear refractive index can be determined. This method is simple, fast and not sensitive to environment noise and vibration. 
Also, it does not need calibration or analysis of fringes patterns.

In addition to the sign of nonlinear refractive index, the value of nonlinear refractive index and absorption coefficient of the samples can also be measured based on these deflected Moiré fringes.

\section{References}

[1] R. A. Ganeev, A. I. Ryasnyansky, S. R. Kamalov, and T. Usmanov, "Nonlinear susceptibilities, absorption coefficients and refractive indices of colloidal metals," J. Phys. D Appl. Phys. 34, 1602-1611 (2001).

[2] J. Zyss, Molecular nonlinear optics: materials, physics, and devices (Academic Press, Boston, 1994).

[3] D. Lupo, Principles and applications of nonlinear optical materials (Chapmann \& Hall, London, 1992), Advanced Materials. 5, 772-773 (1993).

[4] M. Bahae, A. Said, T. Wei, D. Hagan, and E. Van Stryland, "Sensitive measurement of optical nonlinearities using a single beam," IEEE J. Quantum Elect. 26, 760-769 (1990).

[5] M. Sheik-Bahae, A. A. Said, and E. W. Van Stryland, "Highsensitivity, single-beam n2 measurements," Opt. Lett. 14, 955-957 (1989).

[6] M. Sheik-Bahae, J. Wang, R. DeSalvo, D. J. Hagan, and E. W Van Stryland, "Measurement of nondegenerate nonlinearities using a two-color Z scan," Opt. Lett. 17, 258-260 (1992).

[7] M. SheikBahae, A. Said, D. J. Hagan, M. J. Soileau, E. W. Van Stryland, "Nonlinear refraction and optical limiting in thick media," Opt. Eng. 30, 1228-1235 (1991).

[8] S. S. Lin, "Optical properties of $\mathrm{TiO}_{2}$ nanoceramic films as a function of N-Al co-doping," Ceram. Int. 35, 2693-2698 (2009).
[9] S. S. Lin, Y. H. Hung, and S. C. Chen, "Optical properties of $\mathrm{TiO}_{2}$ thin films deposited on polycarbonate by ion beam as-sisted evaporation," Thin Solid Films 517, 4621-4625 (2009).

[10] S. S. Lin, Y. H. Hung, and S. C. Chen, "The properties of $\mathrm{TiO}_{2}$ nanoceramic films prepared by electron beam evaporation," J. Nanosci. Nanotechno. 9, 3599-3605 (2009).

[11] S. S. Lin, S. C. Chen, and Y. H. Hung, " $\mathrm{TiO}_{2}$ nanoceramic films prepared by ion beam assisted evaporation for optical application," Ceram. Int. 35, 1581-1586 (2009).

[12] S. S. Lin, and D. K. Wu, "Enhanced optical properties of $\mathrm{TiO}_{2}$ nanoceramic films by oxygen atmosphere," J. Nanosci. Nanotechno. 10, 1099-1104 (2010).

[13] S. Rasouli, and K. Jamshidi-Ghaleh, "Erratum to' Nonlinear refraction measurements of materials using the moiré deflec-tometry," Opt. Commun. 284, 1481-1482 (2011).

[14] Z. Karny, and 0. Kafri, "Refractive-index measurements by Moiré deflectometry," Appl. Optics 21, 3326-3328 (1982).

[15] 0. Kafri, and I. Glatt, The Physics of Moiré metrology (Wiley, New York, 1989).

[16] K. J. Ghaleh, and N. Mansour, "Nonlinear refraction measurements of materials using the moiré deflectometry," Opt. Commun. 234, 419-425 (2004).

[17] S. Rasouli, H. Chasemi, M. T. Tavassoly, and H. R. Khalesifard, “Application of "parallel" moiré deflectometry and the single beam $Z$-scan technique in the measurement of the nonlinear refractive index," Appl. Optics 50, 2356-2360 (2011).

[18] R. W. Boyd, Nonlinear Optics(Second Edition, Academic Press, Boston, 2003).

[19] E. Hecht, Optics (Fourth Edition, Addison Wesley, Bonn, 2002). 